Supporting Information for Publication

\title{
The Role of Hydrogen in the Electronic Properties of a-Si:H/c-Si Heterostructures
}

Reza Vatan Meidanshahi, * Dragica Vasileska, and Stephen M. Goodnick School of Electrical, Computer and Energy Engineering, Tempe, AZ, 85281, USA

E-mail: rvatanme@asu.edu

A zip file, that contains the xyz coordinations of the most stable configuration of a-Si:H/c-Si at different $\mathrm{H}$ concentrations and the second, third and forth stable configurations of a-Si:H/c-Si at $5.88 \% \mathrm{H}$ atomic concentration, is included. Naming convention is as follow. Label of "csi_asihn.xyz" stands for the xyz coordinations of the most stable configuration of a-Si:H/c-Si heterojunction where there are "n" number of $\mathrm{H}$ atoms in the structure. Label of "csi_asih8_nth.xyz" stands for the xyz coordinates of the "nth" stable configuration of a-Si:H/c-Si heterojunction where $\mathrm{H}$ atomic concentration is $5.88 \%$. 